JOURNAL OF INTEGRAL EQUATIONS

AND APPLICATIONS

Volume 24, Number 1, Spring 2012

\title{
POSITIVE SOLUTIONS FOR A SYSTEM OF NONLINEAR HAMMERSTEIN INTEGRAL EQUATIONS AND APPLICATIONS
}

\author{
JIAFA XU AND ZHILIN YANG \\ Communicated by Paul Martin
}

\begin{abstract}
This paper deals with the existence and multiplicity of positive solutions for the system of nonlinear Hammerstein integral equations

$$
\left\{\begin{array}{l}
u(x)=\int_{0}^{1} k(x, y) f_{1}(y, u(y), v(y), w(y)) \mathrm{d} y, \\
v(x)=\int_{0}^{1} k(x, y) f_{2}(y, u(y), v(y), w(y)) \mathrm{d} y \\
w(x)=\int_{0}^{1} k(x, y) f_{3}(y, u(y), v(y), w(y)) \mathrm{d} y .
\end{array}\right.
$$

We use concave functions to characterize growing and interacting behaviors of our nonlinearities so that $f_{1}, f_{2}, f_{3}$ cover three cases: the first with all superlinear, the second with all sublinear, and the last with two superlinear and the other sublinear. Based on a priori estimates achieved by using Jensen's integral inequality, we use fixed point index theory to establish our main results. As an application, we use our main results to establish the existence and multiplicity of positive solutions for a system of $n$th order boundary value problems for nonlinear ordinary differential equations.
\end{abstract}

1. Introduction. In this paper we study the existence and multiplicity of positive solutions for the following system of nonlinear Hammerstein integral equations

$$
\left\{\begin{array}{l}
u(x)=\int_{0}^{1} k(x, y) f_{1}(y, u(y), v(y), w(y)) \mathrm{d} y \\
v(x)=\int_{0}^{1} k(x, y) f_{2}(y, u(y), v(y), w(y)) \mathrm{d} y \\
w(x)=\int_{0}^{1} k(x, y) f_{3}(y, u(y), v(y), w(y)) \mathrm{d} y
\end{array}\right.
$$

2010 AMS Mathematics subject classification. Primary 45G15, 45M20, 47H07, 47H11, 34A34, 34B18.

Keywords and phrases. System of nonlinear Hammerstein integral equations, positive solution, fixed point index, concave function, $n$th order boundary value problem.

Received by the editors on April 1, 2010, and in revised form on June 3, 2010.

DOI:10.1216/JIE-2012-24-1-131 Copyright (C2012 Rocky Mountain Mathematics Consortium 
where $k \in C\left([0,1] \times[0,1], \mathbf{R}_{+}\right), f_{1}, f_{2}, f_{3} \in C\left([0,1] \times \mathbf{R}_{+}^{3}, \mathbf{R}_{+}\right)\left(\mathbf{R}_{+}:=\right.$ $[0,+\infty))$. Note that, by a positive solution of $(1.1)$, we mean a triple of continuous functions $(u, v, w)$ defined on $[0,1]$ that solve (1.1) and are all nonnegative, with at least one of them not vanishing identically on $[0,1]$.

The solvability of systems of coupled nonlinear integral equations has received a great deal of attention in the literature. For more details of recent development in this direction, we refer the reader to $[\mathbf{5}, \mathbf{9}-\mathbf{1 2}$, 15-18, 21-24] and the references cited therein. In particular, Yang and Donal O'Regan [22] considered the system of Hammerstein integral equations

$$
\left\{\begin{array}{l}
u(x)=\int_{G} k(x, y) f(y, u(y), v(y)) \mathrm{d} y \\
v(x)=\int_{G} k(x, y) g(y, u(y), v(y)) \mathrm{d} y
\end{array}\right.
$$

where $G \subset \mathbf{R}^{n}$ is a bounded closed domain, $k \in C\left(G \times G, \mathbf{R}_{+}\right)$and $f, g \in C\left(G \times \mathbf{R}_{+}^{2}, \mathbf{R}_{+}\right)$. By using the fixed point index theory, they obtained some existence and multiplicity results of positive solutions for system (1.2), where assumptions imposed on the nonlinearities $f$ and $g$ are formulated in terms of spectral radii of associated linear integral operators. To the best of our knowledge, there are only a few papers that deal with systems with three or more equations, see for example $[\mathbf{1}-\mathbf{3}, \mathbf{5}, \mathbf{9}, \mathbf{2 0}]$ and the references cited therein.

In this paper we use concave functions to characterize growing and interacting behaviors of nonlinearities $f_{1}, f_{2}, f_{3}$, so that they cover three cases: the first with all superlinear, the second with all sublinear, and the last with two superlinear and the other sublinear (see Example 1 in Section 3). Based on a priori estimates achieved by using Jensen's integral inequality for concave functions, we use the fixed point index theory to establish our main results. Our work is motivated by [22], but our main results extend and improve the corresponding ones in [22] (see Remark 1 in Section 3). Our main results can be applied to a wide variety of systems of second order and higher order boundary value problems for nonlinear ordinary differential equations, but we confine ourselves to consider, in Section 4, a system of $n$ th-order boundary value problems for nonlinear ordinary differential equations.

This paper is organized as follows. Section 2 contains some preliminary results. The main results are stated and proved in Section 3. 
Finally, in Section 4, we apply our main results to establish the existence and multiplicity of positive solutions for a system of $n$th order boundary value problems for nonlinear ordinary differential equations.

2. Preliminaries. Let $E:=C[0,1],\|u\|:=\max _{x \in[0,1]}|u(x)|$, $P:=\{u \in E: u(x) \geq 0$, for all $x \in[0,1]\}$. Then $(E,\|\cdot\|)$ is a real Banach space and $P$ a cone on $E$. Define

$$
\|(u, v, w)\|:=\max \{\|u\|,\|v\|,\|w\|\},(u, v, w) \in E^{3} .
$$

Note that $E^{3}$ is also a real Banach space under the above norm, and $P^{3}$ is a cone on $E^{3}$.

Define the linear integral operator $L$ by

$$
(L u)(x):=\int_{0}^{1} k(x, y) u(y) \mathrm{d} y .
$$

Then $L: E \rightarrow E$ is a completely continuous positive linear operator. From now on we suppose that the spectral radius of $L$, denoted by $r(L)$, is positive. Furthermore, we assume throughout this paper that the following condition is satisfied:

(H1) There exists a function $e \in C[0,1]$ such that $e$ is almost everywhere positive on $[0,1]$ and satisfies

$$
k(x, y) \geq e(x) k(z, y), \quad \text { for all } x, y, z \in[0,1] .
$$

Define the operators $A_{i}(i=1,2,3)$

$$
A_{i}(u, v, w)(x):=\int_{0}^{1} k(x, y) f_{i}(y, u(y), v(y), w(y)) \mathrm{d} y,(u, v, w) \in P^{3}
$$

and $A$ by

$$
\begin{aligned}
& A(u, v, w)(x) \\
& :=\left(A_{1}(u, v, w)(x), A_{2}(u, v, w)(x), A_{3}(u, v, w)(x)\right), \quad(u, v, w) \in P^{3} .
\end{aligned}
$$

Now $A_{i}: P^{3} \rightarrow P(i=1,2,3)$ and $A: P^{3} \rightarrow P^{3}$ are completely continuous operators if $f_{i} \in C\left([0,1] \times \mathbf{R}_{+}^{3}, \mathbf{R}_{+}^{3}\right)(i=1,2,3)$. Recall that, by a positive solution of (1.1), we mean a triple of functions 
$(u, v, w) \in P^{3} \backslash\{0\}$ that solve (1.1). In our setting, $(u, v, w) \in P^{3}$ is a positive solution of (1.1) if and only if $(u, v, w) \in P^{3} \backslash\{0\}$ is a fixed point of $A$.

Notice we have supposed $r(L)>0$. Then the well-known KreinRutman [14] theorem asserts that there exist two functions $\varphi \in P \backslash\{0\}$ and $\psi \in P \backslash\{0\}$ such that

$$
\int_{0}^{1} k(x, y) \varphi(y) \mathrm{d} y=r(L) \varphi(x), \int_{0}^{1} k(x, y) \psi(x) \mathrm{d} x=r(L) \psi(y)
$$

and

$$
\|\varphi\|=1, \int_{0}^{1} \psi(x) \mathrm{d} x=1
$$

Put

$$
P_{0}:=\left\{u \in P: \int_{0}^{1} \psi(x) u(x) \mathrm{d} x \geq \omega\|u\|\right\},
$$

where $\psi(x)$ is determined by $(2.1),(2.2)$ and $\omega:=\int_{0}^{1} e(x) \psi(x) \mathrm{d} x>0$. Clearly, $P_{0}$ is also a cone on $E$.

Lemma 1 (see [22, Lemma 4]). If $(\mathrm{H} 1)$ is satisfied, then $L(P) \subset P_{0}$.

Lemma 2 (see $[6])$. Let $X$ be a real Banach space and $K$ a cone on $X$. Suppose $\Omega \subset X$ is a bounded open set and $A: \bar{\Omega} \cap K \rightarrow K$ is a completely continuous operator. If there exists $x_{0} \in K \backslash\{0\}$ such that $x-A x \neq \lambda x_{0}$ for all $x \in \partial \Omega \cap K$ and $\lambda \geq 0$, then $i(A, \Omega \cap K, K)=0$ where $i$ indicates the fixed point index on $K$.

Lemma 3 (see [7]). Let $X$ be a real Banach space and $K$ a cone on $X$. Suppose $\Omega \subset X$ is a bounded open set with $0 \in \Omega$ and $A: \bar{\Omega} \cap K \rightarrow K$ is a completely continuous operator. If $x \neq \lambda A x$ for all $x \in \partial \Omega \cap K$ and $\lambda \in[0,1]$, then $i(A, \Omega \cap K, K)=1$.

Lemma 4. Suppose $p: \mathbf{R}_{+} \rightarrow \mathbf{R}_{+}$is a concave function. Then $p$ is nondecreasing on $\mathbf{R}_{+}$, and the following inequality holds:

$$
p(a+b) \leq p(a)+p(b), \quad \text { for all } a, b \in \mathbf{R}_{+} .
$$


In addition, if there exist $0 \leq x_{1}<x_{2}$ such that $p\left(x_{1}\right)=p\left(x_{2}\right)$, then

$$
p(x) \equiv p\left(x_{1}\right), \quad \text { for all } x \geq x_{1} .
$$

Proof. For any $x>x_{2}>x_{1} \geq 0$, we have by the concavity of $p$

$$
p(x) \leq \frac{p\left(x_{2}\right)-p\left(x_{1}\right)}{x_{2}-x_{1}}\left(x-x_{2}\right)+p\left(x_{2}\right) .
$$

This, together with the nonnegativity of $p$, implies $p\left(x_{2}\right) \geq p\left(x_{1}\right)$, which proves that $p$ is nondecreasing on $\mathbf{R}_{+}$. If there exist $0 \leq x_{1}<x_{2}$ such that $p\left(x_{1}\right)=p\left(x_{2}\right)$, then identity (2.5) follows from (2.6) immediately. The proof of (2.4) can be found in [21, Lemma 5]. This completes the proof.

3. Mail results. Let $\lambda_{1}:=1 / r(L)>0$ and $\kappa:=\max _{x, y \in 0,1]} k(x, y)$ $>0$. We now list our hypotheses on $f_{1}, f_{2}, f_{3}$.

(H2) There exist three functions $p, q, s \in C\left(\mathbf{R}_{+}, \mathbf{R}_{+}\right)$such that

(1) $p$ and $q$ are concave on $\mathbf{R}_{+}$,

(2) there exist two constants $c>0$ and $\mu_{1}>1$ such that

$f_{1}(x, u, v, w) \geq p(v)-c, f_{2}(x, u, v, w) \geq q(w)-c, f_{3}(x, u, v, w) \geq s(u)-c$, for all $(x, u, v, w) \in[0,1] \times \mathbf{R}_{+}^{3}$, and

$$
p(\kappa q(\kappa s(u))) \geq \mu_{1} \lambda_{1}^{3} \kappa^{2} u-c, \quad \text { for all } u \in \mathbf{R}_{+} .
$$

(H3) There exist three constants $\eta_{i} \geq 0(i=1,2,3)$ and a sufficiently small $r_{1}>0$ such that $\eta_{1}+\eta_{2}+\eta_{3}<\lambda_{1}$ and

$$
\begin{gathered}
f_{i}(x, u, v, w) \leq \eta_{i}(u+v+w) \\
\text { for all }(x, u, v, w) \in[0,1] \times\left[0, r_{1}\right] \times\left[0, r_{1}\right] \times\left[0, r_{1}\right]
\end{gathered}
$$

(H4) There exist three functions $p_{1}, q_{1}, s_{1} \in C\left(\mathbf{R}_{+}, \mathbf{R}_{+}\right)$such that

(1) $p_{1}$ and $q_{1}$ are concave on $\mathbf{R}_{+}$, 
(2) there exist two constants $r_{2}>0$ and $\mu_{2}>1$ such that

$$
\begin{gathered}
f_{1}(x, u, v, w) \geq p_{1}(v), \\
f_{2}(x, u, v, w) \geq q_{1}(w), \\
f_{3}(x, u, v, w) \geq s_{1}(u), \\
p_{1}\left(\kappa q_{1}\left(\kappa s_{1}(u)\right)\right) \geq \mu_{2} \lambda_{1}^{3} \kappa^{2} u, \\
\text { for all }(x, u, v, w) \in[0,1] \times\left[0, r_{2}\right] \times\left[0, r_{2}\right] \times\left[0, r_{2}\right] .
\end{gathered}
$$

(H5) There exist four constants $\gamma_{i} \geq 0(i=1,2,3)$ and $c>0$ such that $\gamma_{1}+\gamma_{2}+\gamma_{3}<\lambda_{1}$ and

$$
\begin{gathered}
f_{i}(x, u, v, w) \leq \gamma_{i}(u+v+w)+c, \\
\text { for all }(x, u, v, w) \in[0,1] \times \mathbf{R}_{+}^{3}, i=1,2,3 .
\end{gathered}
$$

(H6) $f_{i}(x, u, v, w)(i=1,2,3)$ are nondecreasing in $u, v$ and $w$, and there exists an $N>0$ such that

$$
\int_{0}^{1} k(x, y) f_{i}(y, N, N, N) \mathrm{d} y<N, \quad x \in[0,1], i=1,2,3 .
$$

\section{Example 1. Let}

$$
\begin{aligned}
& f_{1}(x, u, v, w):=\ln \left(u^{2}+v^{2}+w^{2}+1\right) \\
& f_{2}(x, u, v, w)=u^{1.5}+v^{2}+w^{2} \\
& f_{3}(x, u, v, w)=\exp \left(u^{2}+v^{2}+w^{2}\right)-1 .
\end{aligned}
$$

Then (H1) and (H2) hold with $p(v)=\ln (1+v), q(w)=w, r(u)=$ $\exp \left(u^{2}\right)-1$. Obviously, $f_{1}$ grows sublinearly at $+\infty$, whereas $f_{2}$ and $f_{3}$ grow superlinearly at $+\infty$.

We adopt the convention in the sequel that $c_{1}, c_{2}, \ldots$ stand for different positive constants. Let $B_{\rho}:=\left\{(u, v, w) \in E^{3}:\|(u, v, w)\|<\right.$ $\rho\}$ for every $\rho>0$.

Theorem 1. Suppose (H1), (H2) and (H3) are satisfied. Then (1.1) has at least one positive solution $(u, v, w) \in P^{3} \backslash\{0\}$. 


\section{Proof. Let}

$$
\mathscr{M}_{1}:=\left\{(u, v, w) \in P^{3}:(u, v, w)=A(u, v, w)+\lambda(\varphi, \varphi, \varphi), \lambda \geq 0\right\} .
$$

We claim that $\mathscr{M}_{1}$ is bounded. Indeed, if $(u, v, w) \in \mathscr{M}_{1}$, then $u \geq A_{1}(u, v, w), v \geq A_{2}(u, v, w)$ and $w \geq A_{3}(u, v, w)$. By (H2), we have

$$
\begin{gathered}
u(x) \geq \int_{0}^{1} k(x, y) p(v(y)) \mathrm{d} y-c_{1}, \\
v(x) \geq \int_{0}^{1} k(x, y) q(w(y)) \mathrm{d} y-c_{1}, \\
w(x) \geq \int_{0}^{1} k(x, y) s(u(y)) \mathrm{d} y-c_{1},
\end{gathered}
$$

for all $(x, u, v, w) \in[0,1] \times \mathscr{M}_{1}$. Applying Lemma 4 and Jensen's inequality, we obtain

$$
\begin{aligned}
q\left(w(x)+c_{1}\right) & \geq q\left(\int_{0}^{1} k(x, y) s(u(y)) \mathrm{d} y\right) \\
& \geq \kappa^{-1} \int_{0}^{1} k(x, y) q(\kappa s(u(y))) \mathrm{d} y
\end{aligned}
$$

and

$$
\begin{aligned}
p\left(v(x)+c_{1}\right) & \geq p\left(\int_{0}^{1} k(x, y) q(w(y)) \mathrm{d} y\right) \\
& \geq \kappa^{-1} \int_{0}^{1} k(x, y) p(\kappa q(w(y))) \mathrm{d} y \\
& \geq \kappa^{-1} \int_{0}^{1} k(x, y)\left[p\left(\kappa q(w(y))+\kappa q\left(c_{1}\right)\right)-p\left(\kappa q\left(c_{1}\right)\right)\right] \mathrm{d} y \\
& \geq \kappa^{-1} \int_{0}^{1} k(x, y) p\left(\kappa q\left(w(y)+c_{1}\right)\right) \mathrm{d} y-c_{2} .
\end{aligned}
$$

Now (3.2) and (3.3) combined lead to

$$
\begin{aligned}
p\left(v(x)+c_{1}\right) & \geq \kappa^{-1} \int_{0}^{1} k(x, y) p\left(\int_{0}^{1} k(y, z) q(\kappa s(u(z))) \mathrm{d} z\right) \mathrm{d} y-c_{2} \\
& \geq \kappa^{-2} \int_{0}^{1} \int_{0}^{1} k(x, y) k(y, z) p(\kappa q(\kappa s(u(z)))) \mathrm{d} y \mathrm{~d} z-c_{2},
\end{aligned}
$$


and so

$$
\begin{aligned}
p(v(x)) & \geq p\left(v(x)+c_{1}\right)-p\left(c_{1}\right) \\
& \geq \kappa^{-2} \int_{0}^{1} \int_{0}^{1} k(x, y) k(y, z) p(\kappa q(\kappa s(u(z)))) \mathrm{d} y \mathrm{~d} z-c_{2}-p\left(c_{1}\right) .
\end{aligned}
$$

Combining this with (3.1) and (3.5) gives

$$
\begin{aligned}
u(x) & \geq \int_{0}^{1} k(x, y)\left[\kappa^{-2} \int_{0}^{1} \int_{0}^{1} k(y, z) k(z, \tau) p(\kappa q(\kappa s(u(\tau)))) \mathrm{d} z \mathrm{~d} \tau\right. \\
& \left.-c_{2}-p\left(c_{1}\right)\right] \mathrm{d} y-c_{1} \\
& \geq \kappa^{-2} \int_{0}^{1} \int_{0}^{1} \int_{0}^{1} k(x, y) k(y, z) k(z, \tau) p(\kappa q(\kappa s(u(\tau)))) \mathrm{d} y \mathrm{~d} z \mathrm{~d} \tau-c_{3} \\
& \geq \kappa^{-2} \int_{0}^{1} \int_{0}^{1} \int_{0}^{1} k(x, y) k(y, z) k(z, \tau)\left[\mu_{1} \lambda_{1}^{3} \kappa^{2} u(\tau)-c\right] \mathrm{d} y \mathrm{~d} z \mathrm{~d} \tau-c_{3} \\
& \geq \mu_{1} \lambda_{1}^{3} \int_{0}^{1} \int_{0}^{1} \int_{0}^{1} k(x, y) k(y, z) k(z, \tau) u(\tau) \mathrm{d} y \mathrm{~d} z \mathrm{~d} \tau-c_{4} .
\end{aligned}
$$

Multiply both sides of the above by $\psi(x)$, integrate over $[0,1]$ and use (2.1) to obtain

$$
\begin{aligned}
& \int_{0}^{1} \psi(x) u(x) \mathrm{d} x \\
\geq & \mu_{1} \lambda_{1}^{3} \int_{0}^{1} \int_{0}^{1} \int_{0}^{1} \int_{0}^{1} \psi(x) k(x, y) k(y, z) k(z, \tau) u(\tau) \mathrm{d} x \mathrm{~d} y \mathrm{~d} z \mathrm{~d} \tau-c_{4} \\
= & \mu_{1} \int_{0}^{1} \psi(x) u(x) \mathrm{d} x-c_{4}
\end{aligned}
$$

and thus $\int_{0}^{1} \psi(x) u(x) \mathrm{d} x \leq c_{4} /\left(\mu_{1}-1\right)$. Note that, by Lemma 1 , $(u, v, w) \in \mathscr{M}_{1}$ implies $u \in P_{0}, v \in P_{0}$ and $w \in P_{0}$. By (2.3), we obtain

$$
\|u\| \leq \frac{c_{4}}{\omega\left(\mu_{1}-1\right)}, \quad \text { for all }(u, v, w) \in \mathscr{M}_{1} .
$$

Multiply the first inequality in $(3.1)$ by $\psi(x)$, integrate over $[0,1]$, and use (2.1) to obtain

$$
\int_{0}^{1} u(x) \psi(x) \mathrm{d} x \geq \lambda_{1}^{-1} \int_{0}^{1} p(v(x)) \psi(x) \mathrm{d} x-c_{1},
$$


so that

$$
\int_{0}^{1} p(v(x)) \psi(x) \mathrm{d} x \leq \lambda_{1}\left(\|u\|+c_{1}\right) .
$$

Notice that we may assume $v \not \equiv 0$ for any $(u, v, w) \in \mathscr{M}_{1}$ and thus $p(\|v\|)>0$ if $(u, v, w) \in \mathscr{M}_{1}$. Consequently, $v \in P_{0}$ implies

$$
\begin{aligned}
\|v\| & \leq \frac{1}{\omega} \int_{0}^{1} v(x) \psi(x) \mathrm{d} x \\
& \leq \frac{\|v\|}{\omega p(\|v\|)} \int_{0}^{1} \psi(x) \frac{v(x)}{\|v\|} p(\|v\|) \mathrm{d} x \\
& \leq \frac{\|v\|}{\omega p(\|v\|)} \int_{0}^{1} \psi(x) p(v(x)) \mathrm{d} x
\end{aligned}
$$

so that

$$
p(\|v\|) \leq \lambda_{1} \omega^{-1}\left(\|u\|+c_{1}\right), \quad \text { for all }(u, v, w) \in \mathscr{M}_{1} .
$$

By (2) of (H2) we know that $\lim _{v \rightarrow \infty} p(v)=\infty$, and thus there exists a $c_{5}>0$ such that

$$
\|v\| \leq c_{5}, \quad \text { for all }(u, v, w) \in \mathscr{M}_{1} .
$$

A similar argument as used in deriving (3.9) can be used to prove that there exists a $c_{6}>0$ such that

$$
\|w\| \leq c_{6}, \quad \text { for all }(u, v, w) \in \mathscr{M}_{1} .
$$

We find from (3.8), (3.9) and (3.10) that $\mathscr{M}_{1}$ is a bounded set. Consequently, there exists a sufficiently large $R>0$ such that

$$
(u, v, w) \neq A(u, v, w)+\lambda(\varphi, \varphi, \varphi)
$$

for all $(u, v, w) \in \partial B_{R} \cap P^{3}, \quad \lambda \geq 0$.

Now Lemma 2 yields

$$
i\left(A, B_{R} \cap P^{3}, P^{3}\right)=0 .
$$

On the other hand, by (H3) we have

$$
A_{i}(u, v, w) \leq \eta_{i} L(u+v+w), \quad i=1,2,3,
$$


for any $(u, v, w) \in \bar{B}_{r_{1}} \cap P^{3}$. We claim that $I-A$ and $I$ are homotopic on $\partial B_{r_{1}} \cap P^{3}$. It suffices to prove

$$
(u, v, w) \neq \lambda A(u, v, w), \forall(u, v, w) \in \partial B_{r_{1}} \cap P^{3}, \quad \lambda \in[0,1] .
$$

Suppose the contrary. Then there would exist some $\left(u_{0}, v_{0}, w_{0}\right) \in$ $\partial B_{r_{1}} \cap P^{3}$ and $\lambda \in[0,1]$ such that

$$
\begin{aligned}
u_{0} & =\lambda A_{1}\left(u_{0}, v_{0}, w_{0}\right), \\
v_{0} & =\lambda A_{2}\left(u_{0}, v_{0}, w_{0}\right), \\
w_{0} & =\lambda A_{3}\left(u_{0}, v_{0}, w_{0}\right) .
\end{aligned}
$$

It follows from (H3) and (3.14) that

$$
\begin{aligned}
u_{0} & \leq \eta_{1} L\left(u_{0}+v_{0}+w_{0}\right), \\
v_{0} & \leq \eta_{2} L\left(u_{0}+v_{0}+w_{0}\right), \\
w_{0} & \leq \eta_{3} L\left(u_{0}+v_{0}+w_{0}\right),
\end{aligned}
$$

and hence

$$
u_{0}+v_{0}+w_{0} \leq\left(\eta_{1}+\eta_{2}+\eta_{3}\right) L\left(u_{0}+v_{0}+w_{0}\right) .
$$

By successive iteration, we obtain

$$
u_{0}+v_{0}+w_{0} \leq\left(\eta_{1}+\eta_{2}+\eta_{3}\right)^{n} L^{n}\left(u_{0}+v_{0}+w_{0}\right)
$$

for every positive integer $n$. Since $\eta_{1}+\eta_{2}+\eta_{3}<\lambda_{1}$, we have $\left(\eta_{1}+\eta_{2}+\eta_{3}\right)^{n} L^{n}\left(u_{0}+v_{0}+w_{0}\right) \rightarrow 0(n \rightarrow \infty)$. Equation (3.16) then implies $u_{0}=v_{0}=w_{0}=0$, contradicting $\left(u_{0}, v_{0}, w_{0}\right) \in \partial B_{r_{1}} \cap P^{3}$. As a result, (3.13) is true. Now Lemma 3 yields

$$
i\left(A, B_{r_{1}} \cap P^{3}, P^{3}\right)=1 .
$$

Combining (3.11) and (3.17) gives

$$
i\left(A,\left(B_{R} \backslash \bar{B}_{r_{1}}\right) \cap P^{3}, P^{3}\right)=0-1=-1 .
$$

Therefore, operator $A$ has at least one fixed point on $\left(B_{R} \backslash \bar{B}_{r_{1}}\right) \cap P^{3}$, and hence (1.1) has at least one positive solution. This completes the proof. 
Remark 1 . In order to make a comparison with the conditions of $[\mathbf{2 2}]$, we list the assumption $\left(\mathrm{H}_{2}\right)$ of $[\mathbf{2 2}]$.

$\left(\mathrm{H}_{2}\right)$ There exist $p \in C\left(\mathbf{R}_{+}, \mathbf{R}_{+}\right)$and $q \in C\left(\mathbf{R}_{+}, \mathbf{R}_{+}\right)$such that

(1) $p$ is concave and strictly increasing on $\mathbf{R}_{+}$,

(2) $\liminf _{v \rightarrow+\infty}[f(x, u, v) / p(v)]>0, \liminf _{u \rightarrow+\infty}[g(x, u, v) / q(u)]>$ 0 uniformly with respect to $(x, u) \in G \times \mathbf{R}_{+}$and $(x, v) \in G \times \mathbf{R}_{+}$, respectively,

(3) $\lim _{u \rightarrow+\infty}[p(c q(u)) / u]=+\infty$, for all $c>0$.

In our Theorem 1, the counterpart of (3) is $\liminf _{u \rightarrow+\infty}[p(\kappa q(\kappa s(u))) /$ $u]>1$, which considerably weakens (3). In addition, we do not require that $p$ be strictly increasing.

Theorem 2. Suppose (H1), (H4) and (H5) are satisfied. Then (1.1) has at least one positive solution $(u, v, w) \in P^{3} \backslash\{0\}$.

Proof. By (H4), we have

$$
\begin{gathered}
A_{1}(u, v, w)(x) \geq \int_{0}^{1} k(x, y) p(v(y)) \mathrm{d} y, \\
A_{2}(u, v, w)(x) \geq \int_{0}^{1} k(x, y) q(w(y)) \mathrm{d} y, \\
A_{3}(u, v, w)(x) \geq \int_{0}^{1} k(x, y) s(u(y)) \mathrm{d} y, \\
\text { for all }(u, v, w) \in \bar{B}_{r_{2}} \cap P^{3}, \text { for all } x \in[0,1] .
\end{gathered}
$$

We claim

$$
(u, v, w) \nsupseteq A(u, v, w), \quad \text { for all }(u, v, w) \in \partial B_{r_{2}} \cap P^{3} .
$$

If the claim is false, there would exist $\left(u_{0}, v_{0}, w_{0}\right) \in \partial B_{r_{2}} \cap P^{3}$ such that $u_{0} \geq A_{1}\left(u_{0}, v_{0}, w_{0}\right), v_{0} \geq A_{2}\left(u_{0}, v_{0}, w_{0}\right)$ and $w_{0} \geq A_{3}\left(u_{0}, v_{0}, w_{0}\right)$. In view of (3.18), we obtain

$$
\begin{aligned}
& u_{0}(x) \geq \int_{0}^{1} k(x, y) p_{1}\left(v_{0}(y)\right) \mathrm{d} y, \\
& v_{0}(x) \geq \int_{0}^{1} k(x, y) q_{1}\left(w_{0}(y)\right) \mathrm{d} y,
\end{aligned}
$$




$$
w_{0}(x) \geq \int_{0}^{1} k(x, y) s_{1}\left(u_{0}(y)\right) \mathrm{d} y, \quad \text { for all } x \in[0,1] .
$$

Note that $p_{1}$ and $q_{1}$ are concave. Now Jensen's inequality implies

$$
\begin{aligned}
p_{1}\left(v_{0}(x)\right) & \geq p_{1}\left(\int_{0}^{1} k(x, y) q_{1}\left(w_{0}(y)\right) \mathrm{d} y\right) \\
& \geq \kappa^{-1} \int_{0}^{1} k(x, y) p_{1}\left(\kappa q_{1}\left(w_{0}(y)\right) \mathrm{d} y,\right.
\end{aligned}
$$

and

$$
\begin{aligned}
q_{1}\left(w_{0}(x)\right) & \geq q_{1}\left(\int_{0}^{1} k(x, y) s_{1}\left(u_{0}(y)\right) \mathrm{d} y\right) \\
& \geq \kappa^{-1} \int_{0}^{1} k(x, y) q_{1}\left(\kappa s_{1}\left(u_{0}(y)\right) \mathrm{d} y .\right.
\end{aligned}
$$

By (3.20)-(3.22) and (H4), we obtain

$$
\begin{aligned}
u_{0}(x) \geq & \int_{0}^{1} k(x, y) \\
& \times\left[\kappa^{-1} \int_{0}^{1} k(y, z) p_{1}\left(\int_{0}^{1} k(z, \tau) q_{1}\left(\kappa s_{1}\left(u_{0}(\tau)\right) \mathrm{d} \tau\right)\right) \mathrm{d} z\right] \mathrm{d} y \\
\geq & \kappa^{-2} \int_{0}^{1} \int_{0}^{1} \int_{0}^{1} k(x, y) k(y, z) k(z, \tau) p_{1}\left(\kappa q_{1}\left(\kappa s_{1}\left(u_{0}(\tau)\right)\right)\right) \mathrm{d} y \mathrm{~d} z \mathrm{~d} \tau \\
\geq & \mu_{2} \lambda_{1}^{3} \int_{0}^{1} \int_{0}^{1} \int_{0}^{1} k(x, y) k(y, z) k(z, \tau) u_{0}(\tau) \mathrm{d} y \mathrm{~d} z \mathrm{~d} \tau .
\end{aligned}
$$

Multiply both sides of the above by $\psi(x)$, integrate over $[0,1]$ and use (2.1) to obtain

$$
\int_{0}^{1} u_{0}(x) \psi(x) \mathrm{d} x \geq \mu_{2} \int_{0}^{1} u_{0}(x) \psi(x) \mathrm{d} x
$$

so that $\int_{0}^{1} u_{0}(x) \psi(x) \mathrm{d} x=0$, and thus $u_{0}(x) \equiv 0$. By (3.20), we have

$$
\int_{0}^{1} k(x, y) p\left(v_{0}(y)\right) \mathrm{d} y=0
$$


so that $p\left(v_{0}(x)\right) \equiv 0$. This, along with Lemma 4 , implies $v_{0} \equiv 0$. A similar argument shows $w_{0} \equiv 0$. We then have $u_{0}=v_{0}=w_{0}=0$, contradicting $\left(u_{0}, v_{0}, w_{0}\right) \in \partial B_{r_{2}} \cap P^{3}$. As a result, (3.19) is true and

$(u, v, w) \neq A(u, v, w)+\lambda(\varphi, \varphi, \varphi), \quad$ for all $(u, v, w) \in \partial B_{r_{2}} \cap P^{3}, \lambda \geq 0$.

Now Lemma 2 yields

$$
i\left(A, B_{r_{2}} \cap P^{3}, P^{3}\right)=0 .
$$

On the other hand, we have by (H5)

$A_{i}(u, v, w) \leq \gamma_{i} L(u+v+w)+c_{7}, \quad$ for all $(u, v, w) \in P^{3}, i=1,2,3$.

Let

$$
\mathscr{M}_{2}:=\{(u, v, w) \in P \times P \times P:(u, v, w)=\lambda A(u, v, w), 0 \leq \lambda \leq 1\} .
$$

We claim that $\mathscr{M}_{2}$ is bounded. Indeed, for any $(u, v, w) \in \mathscr{M}_{2}$, we have by $(3.26)$,

$$
u+v+w \leq \gamma L(u+v+w)+3 c_{7}
$$

where $\gamma:=\gamma_{1}+\gamma_{2}+\gamma_{3}<\lambda_{1}$. By successive iteration, we obtain

$$
u+v+w \leq \gamma^{n} L^{n}(u+v+w)+\sum_{i=0}^{n-1} \gamma^{i} L^{i}\left(3 c_{7}\right) .
$$

for every $n \geq 1$. This, together with $r(\gamma L)<1$, implies $\gamma^{n} L^{n}(u+v+$ $w) \rightarrow 0$ as $n \rightarrow \infty$ and $u+v+w \leq 3(I-\gamma L)^{-1} c_{7}$, where $(I-\gamma L)^{-1}$ denotes the inverse operator of $I-\gamma L$. The boundedness of $\mathscr{M}_{2}$ has thus been proven. Taking a sufficiently large $R>r_{2}$, we have

$$
(u, v, w) \neq \lambda A(u, v, w), \quad(u, v, w) \in \partial B_{R} \cap P^{3}, \lambda \in[0,1] .
$$

Now Lemma 3 implies

$$
i\left(A, B_{R} \cap P^{3}, P^{3}\right)=1 .
$$


Combining (3.25) and (3.29), we obtain

$$
i\left(A,\left(B_{R} \backslash \bar{B}_{r_{2}}\right) \cap P^{3}, P^{3}\right)=1-0=1 .
$$

Therefore, the operator $A$ has at least one fixed point on $\left(B_{R} \backslash \bar{B}_{r_{2}}\right) \cap P^{3}$, and hence (1.1) has at least one positive solution. This completes the proof.

Theorem 3. Suppose (H1), (H2), (H4) and (H6) are satisfied. Then (1.1) has at least two positive solutions in $P^{3} \backslash\{0\}$.

Proof. By (H6), we have for $i=1,2,3$,

$$
A_{i}(u, v, w)(x) \leq \int_{0}^{1} k(x, y) f_{i}(y, N, N, N) \mathscr{M} y<N
$$

for all $(u, v, w) \in \partial B_{N} \cap P^{3}, x \in[0,1]$, and $i=1,2,3$. This implies

$$
\|A(u, v, w)\|<\|(u, v, w)\|,
$$

and thus

$$
(u, v, w) \neq \lambda A(u, v, w)
$$

for all $(u, v, w) \in \partial B_{N} \cap P^{3}, 0 \leq \lambda \leq 1$. Now Lemma 3 implies

$$
i\left(A, B_{N} \cap P^{3}, P^{3}\right)=1 .
$$

On the other hand, by (H2) and (H4), we may take $R>N$ and $r_{2} \in(0, N)$ so that (3.11) and (3.25) hold (see the proofs of Theorems 1 and 2). Combining (3.11), (3.25) and (3.31), we conclude

$$
i\left(A,\left(B_{R} \backslash \bar{B}_{N}\right) \cap P^{3}, P^{3}\right)=0-1=-1,
$$

and

$$
i\left(A,\left(B_{N} \backslash \bar{B}_{r_{2}}\right) \cap P^{3}, P^{3}\right)=1-0=1 .
$$

Consequently, $A$ has at least two fixed points, one on $\left(B_{R} \backslash \bar{B}_{N}\right) \cap P^{3}$ and the other on $\left(B_{N} \backslash \bar{B}_{r_{2}}\right) \cap P^{3}$. Hence, (1.1) has at least two positive solutions in $P^{3} \backslash\{0\}$. This completes the proof. 
4. Applications. Our main results can be applied to a wide variety of systems of boundary value problems for ordinary differential equations of second order and higher order. In this section, we use our main results to establish the existence and multiplicity of positive solutions for a system of $n$ th-order boundary value problems for nonlinear ordinary differential equations. Our concern in this section is with the problem

$$
\left\{\begin{array}{l}
u^{(n)}(x)+f(x, u, v, w)=0 \\
u(0)=u^{\prime}(0)=\cdots=u^{(n-2)}(0)=u(1)=0 \\
v^{(n)}(x)+g(x, u, v, w)=0 \\
v(0)=v^{\prime}(0)=\cdots=v^{(n-2)}(0)=v(1)=0 \\
w^{(n)}(x)+h(x, u, v, w)=0 \\
w(0)=w^{\prime}(0)=\cdots=w^{(n-2)}(0)=w(1)=0
\end{array}\right.
$$

where $n \geq 2, f, g, h \in C\left([0,1] \times \mathbf{R}_{+}^{3}, \mathbf{R}_{+}\right)$. Problem (4.1) is equivalent to the system of nonlinear Hammerstein integral equations

$$
\left\{\begin{array}{l}
u(x)=\int_{0}^{1} k(x, y) f(y, u(y), v(y), w(y)) \mathrm{d} y \\
v(x)=\int_{0}^{1} k(x, y) g(y, u(y), v(y), w(y)) \mathrm{d} y \\
w(x)=\int_{0}^{1} k(x, y) h(y, u(y), v(y), w(y)) \mathrm{d} y
\end{array}\right.
$$

where $k(x, y)$ is the Green's function

$$
k(x, y)= \begin{cases}{\left[(1-y)^{n-1} x^{n-1}-(x-y)^{n-1}\right] /(n-1) !} & 0 \leq y \leq x \leq 1 \\ {\left[(1-y)^{n-1} x^{n-1}\right] /(n-1) !} & 0 \leq x \leq y \leq 1\end{cases}
$$

Note that $k \in C\left([0,1] \times[0,1], \mathbf{R}_{+}\right)$satisfies the relation (see $[\mathbf{1 9}$, Lemma $2.2]$ )

$$
k(x, y) \geq e(x) k(z, y), \quad \text { for all } x, y, z \in[0,1]
$$

where

$$
\begin{aligned}
e(x) & :=\min \left\{\frac{x^{n-1}}{n-1}, \frac{(1-x) x^{n-2}}{n-1}\right\} \\
& = \begin{cases}\left(x^{n-1}\right) /(n-1) & 0 \leq x \leq 1 / 2, \\
{\left[(1-x) x^{n-2} /(n-1)\right]} & 1 / 2 \leq x \leq 1 .\end{cases}
\end{aligned}
$$


This implies that (H1) in Section 2 is satisfied. Simple computations show that

$$
\kappa:=\max _{(x, y) \in[0,1] \times[0,1]} k(x, y)=\frac{(n-1)^{n-1}}{n^{n}(n-2) !} .
$$

Define the completely continuous linear operator $L: E \rightarrow E$ by

$$
(L u)(x):=\int_{0}^{1} k(x, y) u(y) \mathrm{d} y .
$$

It is easy to prove that $r(L)>0$. Applying Theorems 1-3, we obtain the following results:

Theorem 4. Suppose (H2) and (H3) are satisfied. Then system (4.1) has at least one positive solution $(u, v, w) \in\left(C^{n}[0,1]\right)^{3} \cap\left(P^{3} \backslash\{0\}\right)$.

Theorem 5. Suppose (H4) and (H5) are satisfied. Then system (4.10) has at least one positive solution $(u, v, w) \in\left(C^{n}[0,1]\right)^{3} \cap$ $\left(P^{3} \backslash\{0\}\right)$.

Theorem 6. Suppose $(\mathrm{H} 2),(\mathrm{H} 4)$ and $(\mathrm{H} 6)$ are satisfied. Then system (4.1) has at least two positive solutions on $\left(C^{n}[0,1]\right)^{3} \cap\left(P^{3} \backslash\{0\}\right)$.

Acknowledgments. The authors would like to thank the referees for their constructive comments.

\section{REFERENCES}

1. Ravi P. Agarwal, Donal O'Regan and Patricia J.Y.Wong, Constant-sign solutions of systems of higher order boundary value problems with integrable singularities, Math. Comp. Model. 44 (2006), 983-1008.

2. - Constant-sign solutions for systems of singular integral equations of Hammerstein type, Math. Comp.r Model. 50 (2009), 999-1025.

3. - Dynamics of epidemics in homogeneous/heterogeneous populations and the spreading of multiple inter-related infectious diseases: Constant-sign periodic solutions for the discrete model, Nonlinear Anal.: Real World Appl. 8 (2007), 1040-1061.

4. K. Deimling, Nonlinear functional analysis, Springer-Verlag, New York, 1985.

5. D. Franco, G. Infante and Donal O'Regan, Nontrivial solutions in abstract cones for Hammerstein integral systems, Dynam. Contin. Discrete Impuls. Syst. Math. Anal. 14 (2007), 837-850.

6. D. Guo, Nonlinear functional analysis, Science and Technology Press of Shandong, Jinan, 1985 (in Chinese).

7. D. Guo and V. Lakshmikantham, Nonlinear problems in abstract cones, Academic Press, Boston, 1988.

8. D. Guo and J. Sun, Nonlinear integral equations, Shandong Press of Science and Technology, Jinan, 1987 (in Chinese). 
9. J. Henderson and H. Wang, An eigenvalue problem for quasilinear systems, Rocky Mountain J. Math. 37 (2007), 215-228.

10. G. Infante and P. Pietramala, Existence and multiplicity of non-negative solutions for systems of perturbed Hammerstein integral equations, Nonlinear Anal. 71 (2009), 1301-1310.

11. _ Eigenvalues and nonnegative solutions of a system with nonlocal BCs, Nonlinear Stud. 16 (2009), 187-196.

12. P. Kang, J. Xu and Z. Wei, Positive solutions for $2 p$-order and $2 q$-order systems of singular boundary value problems with integral boundary conditions, Nonlinear Anal. 72 (2010), 2767-2786.

13. M.A. Krasnoselskii, Topological methods in the theory of nonlinear integral equations, Pergamon, Oxford, 1964.

14. M.G. Krein and M.A. Rutman, Linear operators leaving invariant a cone in a Banach space, Transl. Amer. Math. Soc. 10 (1962), 199-325.

15. B. Liu, L. Liu and Y. Wu, Positive solutions for singular systems of threepoint boundary value problems, Comput. Math. Appl. 53 (2007), 1429-1438.

16. R. Precup, A vector version of Krasnoselskii's fixed point theorem in cones and positive periodic solutions of nonlinear systems, J. Fixed Point Theory Appl. 2 (2007), 141-151.

17. H. Su, Z. Wei, X. Zhang and J. Liu, Positive solutions of $n$-order and $m$ order multi-point singular boundary value system, Appl. Math. Comp. 188 (2007), $1234-1243$.

18. J. Sun and X. Liu, Computation for topological degree and its applications, J. Math. Anal. Appl. 202 (1996), 785-796.

19. D. Xie, C. Bai, Y. Liu and C. Wang, Positive solutions for nonlinear semipositone nth-order boundary value problems, Electr. J. Qual. Theory Differ. Equat. 7 (2008), 1-12.

20. X. Yang, Existence of positive solutions for $2 \mathrm{~m}$-order nonlinear differential systems, Nonlinear Anal. 61 (2005), 77-95.

21. Z. Yang, Positive solutions to a system of second-order nonlocal boundary value problems, Nonlinear Anal. 62 (2005), 1251-1265.

22. Z. Yang and Donal O'Regan, Positive solvability of systems of nonlinear Hammerstein integral equations, J. Math. Anal. Appl. 311 (2005), 600-614.

23. Z. Yang and J. Sun, Positive solutions of boundary value problems for systems of nonlinear second order ordinary differential equations, Acta Math. Sinica 47 (2004), 111-118 (in Chinese).

24. Z. Zhang, Existence of non-trivial solution for superlinear system of integral equations and its applications, Acta Math. Sinica 15 (1999), 153-162 (in Chinese).

Department of Mathematics, Qingdao Technological University, QingDao, Shandong, China

Email address: jiafaxu@sina.cn, xujiafa292@sina.com

Department of Mathematics, Qingdao Technological University, QingDaO, Shandong, China

Email address: zhilinyang@sina.com, zhilinyang@ymail.com 